\begin{tabular}{|c|c|}
\hline \multirow{3}{*}{ 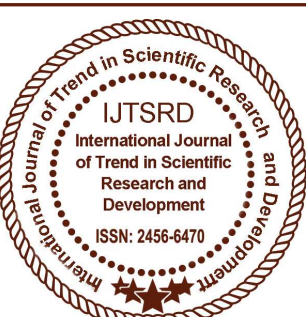 } & $\begin{array}{l}\text { International Journal of Trend in Scientific } \\
\text { Research and Development (IJTSRD) }\end{array}$ \\
\hline & International Open Access Journal \\
\hline & ISSN No: 2456 - 6470 | www.ijtsrd.com | Volume - 2 | Issue -3 \\
\hline
\end{tabular}

\title{
Comparing the effectiveness of Schirmer's test I and Non-invasive tear break-up time (NITBUT) for the assessment of dry eye among University students: A case-control study
}

\author{
Wejdan Salim Matar Al-Maqbali \\ University of Buraimi, Buraimi, Oman
}

\section{ABSTRACT}

Aim: To compare the effectiveness of Schirmer's test I and non-invasive tear break-up time (NITBUT) for the assessment of dry eye among University of Buraimi students.

Methods: This prospective cross-sectional comparative study included 60 students from the University of Buraimi aged between 21-25 years and from three majors. Participants were selected using purposive sampling method. MacMonnies dry eye questionnaire was used to categorize the members as case group and control group. NITBUT followed by Schirmer's test-I was conducted for each participant's only one eye, which was randomly selected. Descriptive statistics was used to evaluate the results and means are used to compare the two tests.

Results: The mean of McMonnies Screening of the overall scores for all participants were 6.45 with an (SD) of 0.4472 . The mean of Schirmer's test $I$ is 25.4667 and $(\mathrm{SD}=7.3904)$, while the Noninvasive TBUT was 5.0666 and $(\mathrm{SD}=1.6020)$.

Conclusions: The study demonstrated a better sensitivity of the NITBUT test compared to Schirmer's test I. However, for borderline case group, there is an inverse correlation between questioners score and NITBUT values as the questioner's show borderline while measurements show the opposite.

Keywords: NITBUT, Schirmer's test I, screening tool, effectiveness, McMonnies dry eye questionnaire

\section{INTRODUCTION}

\section{Background}

Dry eye is one of the common causes which hampers the vision-related quality of life. It associates with symptoms of discomfort, visual disturbance, and tear film instability with acute damage to the ocular surfacel. Accurate and timely diagnosis would be the management key.

The outer portion of the eye involves some structures, each of which has its particular function. 'The ocular surface, tear film, lacrimal glands, and eyelids.' Act as a functional unit to maintain the quality of the refractive surface of the eye; to provide protection for the eye against the environmental conditions. Dry eye is a condition of tear film abnormality. There are three layers of the tear film: an outer lipid layer, a thin aqueous layer in the middle and mucous layer. ${ }^{9}$

As it's a famous ophthalmological case, $73 \%$ of the patients and more show an expansion of this condition $^{2}$. The population of $>40$ years old have a significant percentage of Dry eye syndrome. Although it can affect any race, it is more prevalent in women. A daily life of computers users considers being one of the risk factor ${ }^{1}$.

Various invasive and noninvasive methods are utilized in the assessment of the dry eye. Several techniques are used to evaluate tear film's quantity and quality. And it is a sufficient cognitive comprehension would be very helpful for clinical diagnosis $^{2}$. 
Schirmer test I, tear break-up time (TBUT) are the most popular objective tests used for diagnosis of a dry eye condition. However, neither symptoms or signs always match with the results of these tests ${ }^{8}$

'The McMonnies dry eye questionnaire is considered to be the 'gold standard' for the diagnosis of dry eye symptoms. This is the reason behind selecting this questionnaire for this study as it is considered to be earliest and most widely used screening for dry eye with a sensitivity ranging between $87 \%$ and $98 \%$ and a specificity of between $87 \%$ and $97 \%$. $^{9}$

A dry eye questionnaire considers as a screening instrument with clinic procedure as it correlates with the sensitivity and specificity. This investigation helps to concerned with how the dry eye questionnaire responses should be considered to classify participants into dry eye, marginal dry eye, and non-dry eye groups. ${ }^{11}$ The limitations of 'studies include different age distributions of the population, definitions of dry eye, and methodologies. Studies on the tests of tear function, including Schirmer's test I', tear breakup time test (TBUT) and the nature and circumstances of the environment or the clinic which require more proper preparations.

However, the gap between patient symptoms and the clinical observations exists. Hence, to identify the best assessment method. The proposed study compares the findings of the Schirmer's I and noninvasive TBUT and its associated patient symptoms.

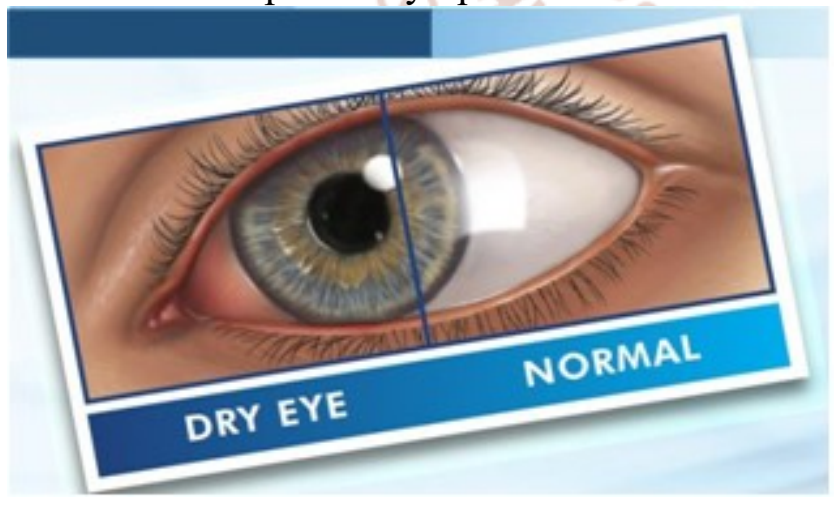

\section{Literature Review}

A study was done by Tung $\mathrm{C}$ et al. 1 to find out the relationships between tear meniscus dimensions and parameters of ocular surface disease in a various tear dysfunction conditions. Shows that Tear meniscus highly correlated with TBUT for all participants. They conclude that in tear dysfunction conditions lower tear volume has a strong relation with the 'corneal epithelial disease in ATD and Sjögren syndrome, conditions with lacrimal gland dysfunction. In contrast, higher tear volume' is correlated with corneal epithelial disease in MGD. Moreover, a study shows only $57 \%$ complain of having symptoms consistent with a diagnosis of DED. Where the TBUT(0.12), Schirmer test (0.09), corneal and conjunctival staining were the same. It was done by Benjamin D. Sullivan et al. 2 to estimate the relevance 'between 'signs and symptoms of dry eye disease (DED) in a clinic- based' population' concluding 'that no consistent correlation was found between the signs and symptoms of DED. Each type of measurement provides various information about the condition of the ocular surface. These results also proved that symptoms alone are insufficient for the diagnosis and management of DED and argue for an assent of clinical signs that better reflect all aspects of the disease. Furthermore, a study finds out that $(75.6 \%)$ patients had abnormal result and $(24.4 \%)$ had average TBUT. While $(9.3 \%)$ eyes with pterygium had Schirmer's test positive and $(90.7 \%)$ eyes had it negative. So they conclude that tear film breakup time test has better and accurate diagnostic value compared to Schirmer's test in determining tear film abnormality in patients with pterygium 3 . These findings may support the ability to detect patients at risk for the corneal epithelial disease. In contrast, a study was done by Cihan Ünlü et al. 4 to compare the diagnostic values of the Schirmer's and tear film breakup time (TBUT) tests, and the Ocular Surface Disease Index (OSDI) in dryeye syndrome approved that There was a significant opposite correlation between the OSDI and TBUT scores. On the other hand, they found that there is no significant relationship existed between the OSDI and Schirmer's test scores and the primary outcomes clarify 'that dry eye is a common issue among computer users. The OSDI questionnaire used together with the TBUT is easily performed and support the diagnosis of dry-eye syndrome'. However, Fermon $\mathrm{S}$ et al. 5 conducted a study about standardizing the Schirmer test I and tear break-up time involving a group of healthy people from a Mexican Ophthalmological Hospital they conclude that in Mexican people, the median of the Schirmer test and break-up time test is shorter than other reports. Among the study, there were no statistical significance in the difference between the genders results. The primary outcomes aim to set a new "normal standard" of the test to classify dry eye syndromes. 'Moreover, a study was done by Monica Alves et al. 6 states that the best collection of 
diagnostic tests for DED was OSDI, TBUT and Schirmer test (sensitivity $100 \%$, specificity $95 \%$ and accuracy $99.3 \%$ ). And the outcomes shows that DED diagnostic test results present a wide range of variability among several conditions'. Vital staining's and TBUT correlated best with one another whereas the best test combination to identify DED was: OSDI/TBUT/ Schirmer. As the study aim 'was a 'compares signs, symptoms and predictive tools used to diagnose dry eye disease (DED) and ocular surface disorders in a different systemic well-defined and non-overlapping diseases.' A study done by B E Kurtul et al. 7 find out that TBUT scores and Schirmer-1 results of the study group were significantly lower than the control group. As the aim of the survey is To identify the effect of vitamin D deficiency on tear break-up time (TBUT) and Schirmer test scores and to estimate their relationship in non-Sjögren dry-eye patients. A study showed the prevalence of dry eye disease assessed by the tear osmolarity test was $27.7 \%$ and the primary outcomes were, the available standard diagnostic tests underestimate the presence of dry eye disease in people with type 2 diabetes. Moreover, they could not recognized tear hyperosmolarity from normal. Tear osmolarity could be considered as the best test for estimating of dry eye disease in people with type 2 diabetes. As the following tests were also performed: Ocular Surface Disease Index (OSDI) questionnaire, Tear Film Breakup Time (TFBUT), Schirmer I test, Rose Bengal and Fluorescein staining. The outcomes of these tests were compared to the tear osmolarity measurement8. Another study was done by Naimah Ebrahim Khan 9 shows a strong positive relation respectively was found between the both eyes. As the aim is to establish normal values for Schirmer tear test and tear break-up time (TBUT) in a South African young adult Black population. The outcomes were. The mean values found in this study for the Schirmer test are the same to those that have been reported in the literature. However, values for TBUT differ from the values that have been previously reported, being higher in some instances and lower in others. These findings for dry eye diagnosis and also contact lens practice in South Africa. One more study was about effect of Duration of Diabetes on Schirmer's Test and Tear Film Break up Time 10. It stated that there is strong negative relation between duration of diabetes and TBUT value. There is a moderate negative relationship between length and Schirmer's value which is estimated to be statistically significant $(p<0.001)$. Diabetes adversely affects the tear film parameters. As the duration of diabetes increases, there is fall in both Schirmer's value and TBUT value.

\section{Rationale for the Study}

Dry eye is one of the common causes which hampers the vision-related quality of life. Various invasive and noninvasive methods are used in the assessment of the dry eye. However, the gap between patient symptoms and the clinical observations exists. Hence to identify the best evaluation methods among the routine tests, the proposed study compares the findings of the Schirmer's I and noninvasive TBUT and its associated patient symptoms.

\section{Aim of Study}

To compare the effectiveness of Schirmer's test I and noninvasive (NITBUT) for the assessment of dry eye among University of Buraimi students.

\section{MATERIALS AND METHODS}

The examinations were conducted at the University of Buraimi's Optometry contact lens lab. The room temperature and level of illumination and instrument positioning are strictly kept under control to limit the confounding variables.

The McMonnies dry eye questionnaire with Scoring scheme that was disrupted among the participants with some modifying in questions according to the culture. Participants were randomly selected, with each person being informed by the procedures and signing written consent to participate. Questionnaires have been a standard tool in the dry eye diagnosis of dry eye; it is considered to be as screening tools. Surveys based on symptoms have been used to screen populations. Dry eye symptom questionnaires were a useful way to classify participants into groups.

The room temperature was 23 degrees, and there was four lights lamp with an intensity of 220 Volt and 26 Watts. The clinic is located on the third floor and supplied by all the instruments that were required for the research. After answering the questionnaire that classified them into case and control groups based on their score. A score of more than 20 considered as case group while less than 20 is the control group.

The measurements started by using Schirmers test strips (Optitech eye care) (Whatman filter paper no.41) which is $5 \mathrm{~mm} \times 35 \mathrm{~mm}$. In the lower fornix near the lateral canthus, the strips were placed away from the cornea and with eyes closed' it kept for 5 
minutes for a randomly selected eye for each student in both groups. As the patients were directed to look forward and to blink frequently during the test.

Followed by Non-invasive TBUT using keratometry (Bausch \& Lomb) to observe the interval between the last blink and the first appearances of the distorted keratometry mires.

The subject was asked to blink three times and then look straight forward, without blinking to take the average. The tear film was observed under the keratometry instrument and time between the last blink and first appearance of the first break in the tear film was recorded with a stopwatch. This procedure was repeated three times on one eye for each participant. A normal tear breakup time (TBUT) is between 15 and 45 seconds and less than 10 seconds is indicative of unstable tear film. Schirmer's result of $10-15 \mathrm{~mm}$ or more is regarded as normal tear production. A value of less than $5 \mathrm{~mm}$ is considered to be suggestive of a dry eye.

\section{SAMPLE SIZE}

Open Epi software was used to calculate the minimum sample size. Keeping the confidence interval $(1-a)=$ $95 \%$ and absolute precision (d) of 0.08 , the estimated sample size is calculated as 60 . It involved 30 females in each group. A self-designed and validated questionnaire was used to classify them sort them into the groups. The survey collected patient responses on the grading of their symptoms such as itching, burning, watering and visual fluctuations using a Likert scale.

\section{INCLUSION CRITERIA}

The study included all the participant with and without dry eye symptoms which are not undergoing any systemic or ocular treatment.

\section{EXCLUSION CRITERIA}

Students are wearing contact lenses, previous ocular disorders, intraocular surgery, nasolacrimal duct obstruction, or who use topical ophthalmic or systemic medications were excluded from the study.

\section{Data Analysis}

The questionnaire based responses identifying the association between case and control group was analyzed using chi - square test. Multiple regression analysis was used to analyze the most common dry eye symptoms. For each parametric value mean and the standard deviation was calculated. Microsoft Excel (2013) were used to store, analyze the data and to carry descriptive statistics.

\section{RESULTS}

\section{DEMOGRAPHIC DETAILS}

The population sample consisted of 60 participants from three majors (optometry, nursing, and engineering )resulting in 60 eyes were examined. The age of the participants ranged from 21 to 25 years, with a mean of 23 years and standard deviation $(\mathrm{SD}=$ 1.082). The optometry students were more than $50 \%$ of the sample population (figure 1). Schirmer's test I values $(\mathrm{n}=60)$ have a total mean 25.4667 and $(\mathrm{SD}=$ 7.3904 ). While the Noninvasive TBUT were 5.0666 and $(\mathrm{SD}=1.6020)$.

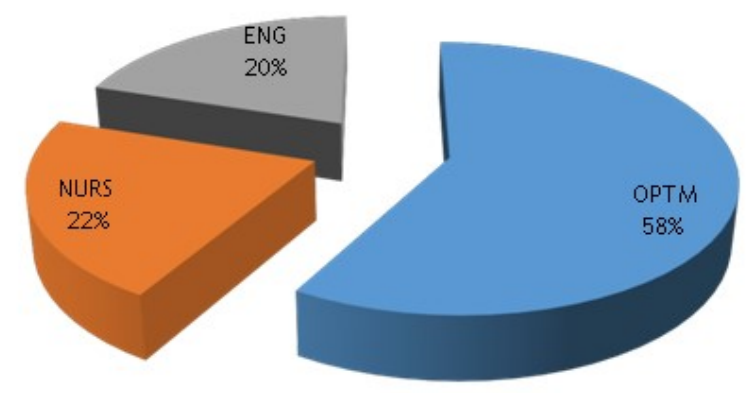

Figure 1: The number of participants according to majors

Table 1 shows that number of engineering students having moderate dry eye among the other majors. Hence the engineering students use the computers for long hours. 
International Journal of Trend in Scientific Research and Development (IJTSRD) ISSN: 2456-6470

Table 1: Major wise comparison of Schirmer's test I and NITBUT among participants

\begin{tabular}{|c|c|c|c|c|}
\hline & $\begin{array}{c}\text { Schirmer's } \\
\text { test I }\end{array}$ & Mean & NITBUT & Mean \\
\hline OPTM (n=35) & & & & \\
\hline less than 5 & $1(2.9 \%)$ & 0.114 & $21(60 \%)$ & 1.93 \\
\hline 5 to 10 & $3(8.8 \%)$ & 0.742 & $7(20 \%)$ & 1.295 \\
\hline more than 10 & $31(88.5 \%)$ & 26.5 & $7(20 \%)$ & 3.09 \\
\hline NURS (n=13) & & & & \\
\hline less than 5 & 0 & 0 & $8(61.5 \%)$ & 2.06 \\
\hline Between 5-10 & $1(7.6 \%)$ & 0.692 & $5(38.4 \%)$ & 2.03 \\
\hline more than 10 & $12(92.3 \%)$ & 25.3 & 0 & 0 \\
\hline ENG ( n=12) & & & & \\
\hline less than 5 & $3(25 \%)$ & 0.83 & $7(58.3 \%)$ & 2.04 \\
\hline Between 5-10 & $3(25 \%)$ & 1.5 & $5(41.6 \%)$ & 3.33 \\
\hline more than 10 & $7(58.3 \%)$ & 17.3 & 0 & 0 \\
\hline
\end{tabular}

Table 2 show a McMonnies Screening of 60 female subjects were involved in the study. Each group consists of 30 participants based on symptoms to screen the populations. The mean for the overall scores for all participants were 6.45 with an (SD) of 0.4472. The maximum score for all the participant was 16 and minimum was 0.

The noninvasive Tear breaks up test values for all the participants varies from 2 seconds to 20 seconds for the participants. The Schirmer's test I values was between $3 \mathrm{~mm}$ to $35 \mathrm{~mm}$ among the participants.
The number of participants having NITBUT values less than 5, were high compared to questioner's sore and Schirmer's I test values for (less than 5) as 36 cases were showing a dry eye. In contrast, it was low for (5-10) range. However, there is an inverse correlation between questioners score and NITBUT values as the questioner's show borderline while measurements show the opposite. According to the questionnaire, a score of over 20 is indicative of the dry eye, while a total score of between 10 and 20 is suggestive of borderline dry eye disease. On the other hand, the Schirmer test I and NITBUT values of (less than 5) consider as dry eye, (between 5-10) marginal dry eye, (between 11-20) a borderline dry eye and (more than 20) as a normal condition.

Table 2: Descriptive details showing the questionnaire score,

NITBUT and Schirmer's test I values for all the participants

\begin{tabular}{|c|c|c|c|}
\hline & Q Score & NITBUT & Schirmer's test I \\
\hline Less than 5 & $15(25 \%)$ & $36(60 \%)$ & $3(5 \%)$ \\
\hline Between 5-10 & $38(63.33 \%)$ & $17(28.3 \%)$ & $7(11.6 \%)$ \\
\hline Between 11-15 & $6(10 \%)$ & $5(8.33 \%)$ & $6(10 \%)$ \\
\hline Between 16-20 & $1(1.66 \%)$ & $1(1.66 \%)$ & 0 \\
\hline More than 20 & 0 & $1(1.66 \%)$ & $44(73.33 \%)$ \\
\hline
\end{tabular}

\section{DISCUSSION}

The purpose of this study was to compare effectiveness of Schirmer's test I and non- invasive (NITBUT) for the assessment of dry eye among University of Buraimi students. By identifying the comparing between the Schirmer's test, NITBUT used and the symptoms. However, the gap between patient symptoms and the clinical observations exists. As far as we searched the literature, this study was the first to compare between Schirmer's test I and NITBUT all the others compared between Schirmer test I or II and the invasive techniques. The study findings show that there is an inverse correlation between questioners score and NITBUT values as the questioner's grading reflects borderline dry eye, while measurements show the opposite. Also, the number of participants having NITBUT values less than 5, were high compared to questioner's sore. For the 
convenient randomized sampling, participants aged 21-25 years were chosen for this study. The gender of participants in this study is different from the other selected studies that have shown both sexes for the assessment of tear values. The age of participants in this study is different from the range of the participants in other studies. Some researchers preferred to group members according to various age brackets; they have either included participants with a mean age of $55.8 \pm 10.33$ years old 8 or 48.8 years - \pm 14.1 years from both genders 6 . Hence the study can be explored with different age groups within Omani population.

A study identified TBUT is having good sensitivity compared to the Schirmer's test and considered to be an important diagnostic tool for detecting tear deficiency in eyes with pterygium 3 . Similar results are also identified by a study done by Cihan Ünlü et al. 4 .

However, a study done by Dr. Rashmi S et al. 10 found that Schirmer's test and rose bengal test have more diagnostic value in people with diabetes as compared to TBUT. This could be due to the different age group considered for the study. The advantage of having the same gender is an accurate way to compare between the Schirmer's test I and NITBUT for the assessment of dry eye. Hence dry eye disease affects women twice as often as men 12 .

The study showed that the mean values obtained for NITBUT in the current study were marginally lower than Schirmer's test I that is given in table 1 . However, there is an inverse correlation between questioners score and NITBUT values as the questioner's grading reflects borderline dry eye while measurements indicate the opposite that is shown in Table 2. In contrast, a study done by Khan Naimah Ebrahim 9 observed that Schirmer value for was marginally higher than the TBUT values.

A study done by Fermon $\mathrm{S}$ et al. used Benitez del Castillo questionnaire five while other studies 9 including ours used MacMonnies dry eye questionnaire with Scoring scheme as it is a useful screening tool for dry eye assessments.

Schirmer test I and NITBUT values of (less than 5) consider as dry eye and (more than 10) as a normal condition that has been widely accepted among the previous studies.5

The study showed that NITBUT has a better measurement on the population used than those found in the literature who depend on the using of invasive technique. The Schirmer and the non-invasive tear break up time are very easy to test, inexpensive and do not represent any risk for the patient. However, our study did not find the two test as reliable in the assessment of dry eye.

It might be interesting to perform further studies in different types of population that might support the result of this study. Future studies should involve a larger population sample including all the students from various primary and occupation. The age of the participants should be expanded and include all age groups among the students, those who are younger and older than those included in this study. Furthermore, factors such as contact lens wear, age, systemic diseases, gender, and environmental factors such as air conditioners, room illumination, and room temperature should be monitor accurately.

\section{CONCLUSION}

The study demonstrated a better sensitivity of the NITBUT test compared to Schirmer's test I. Thus; it could be used as an initial screening tool to detect tear film instability in patients. However, the study identified the inverse relationship of TBUT reading with the Macmonnies questionnaire score. Using higher sample size and involving both genders can be helpful in further research.

\section{REFERENCES}

1. Tung CI, Perin AF, Gumus K, Pflugfelder SC.Tear Meniscus Dimensions in Tear Dysfunction and Their Correlation With Clinical Parameters. Am J Ophthalmol 2014; 157:301-10

2. Sullivan BD,Crews LA, Messmer EM,Foulks GN,Nichols KK, GerdGeerling PB ,et al. Correlations between commonly used objective signs and symptoms for the diagnosis of dry eye disease: clinical implications 2012; 92:161-6.

3. Rahman A, Fasih U, Ul-Huda W, Shaikh A, Yahya K. Comparison of Schirmers test and tear film breakup time test to detect tear film abnormalities in patients with Pterygium. Journal Of Pakistan Medical Association 2012; 62:121416.

4. Ünlü C, Güney E, Lkay B ,Akçay S, Akçalı G ,Erdogan G, et al. Comparison of ocular-surface disease index questionnaire, tear film break-up time, and Schirmer tests for the evaluation of the 
International Journal of Trend in Scientific Research and Development (IJTSRD) ISSN: 2456-6470

tear film in computer users with and without dryeye symptomatology. Clinical Ophthalmology 2012;6:1303-6.

5. Fermon S, Ball S, Paulin JM, Davila R, Guttman S. Schirmer I Test and Break-Up Time Test Standardization in Mexican Population Without Dry Eye. Mexicana de Oftalmología 2010 ; 84:228-32.

6. Alves M, Reinach PS, Paula JS, e Cruz AA, Bachette L, Faustino J, Aranha FP, Vigorito A, de Souza CA, Rocha EM. Comparison of diagnostic tests in distinct well-defined conditions related to dry eye disease. PloS one. 2014 May 21;9(5):e97921.

7. Kurtul BE, Özer PA, Aydinli MS. The association of vitamin D deficiency with tear break-up time and Schirmer testing in non-Sjögren dry eye. Eye. 2015 Aug 1;29(8):1081-4.

8. Najafi L, Malek M, Valojerdi AE, Khamseh ME, Aghaei H. Dry eye disease in type 2 diabetes mellitus; comparison of the tear osmolarity test with other common diagnostic tests: a diagnostic accuracy study using STARD standard. Journal of
Diabetes \& Metabolic Disorders. 2015 Apr 29;14(1):39.

9. Khan NE. Schirmer Tear Test 2 and Tear BreakUp Time values in a South African young black adult population.Unversity of Kwazulu-Natali 2012:1-110.

10. Rashmi S, Adappa P, Hegde V, Jain R, Anupama B. Impact of Duration of Diabetes on Schirmer's Test and Tear Film Break up Time: IOSR-JDMS 2016; 15(6):74-2.

11. McMonnies C, Ho A ,Wakefield D. Basic Science and Clinical Relevance Lacrimal Gland, Tear Film, and Dry Eye Syndromes 2. Optimum Dry Eye Classification Using Questionnaire Responses.1st ed. Australia: University of new south wales; 1998: 835.

12. Schaumberg DA, Uchino M, Christen WG, Semba RD, Buring JE, Li JZ (2013) Patient Reported Differences in Dry Eye Disease between Men and Women: Impact, Management, and Patient Satisfaction. PLoS ONE 8(9): e76121. doi:10.1371/journal.pone.0076121.

\section{APPENDICES (A):}

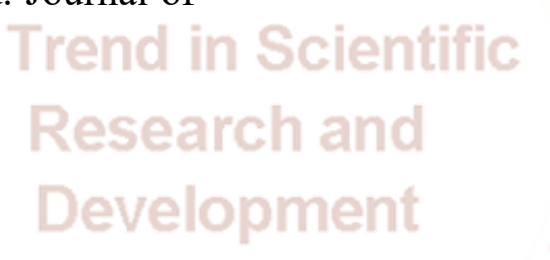

College of Health Sciences (Optometry)

Sequence Number :

Date:

Date of Birth:

Sex: M / F (Circle)

Dry eye occurs when there is in sufficient amount of tears in your eye necessary for maintaining moisture and proper surface nourishment. There are several for the lack of tears. The feeling of dry eyes, sometimes inconvenient as it is possible to be accompanied by the sense of burning or twitching in the eye. Many times we face such situations. For example, while exposure to air-conditioned or during TV watching continuously for several hours. Our study aims at understanding the diagnostic differences on patient symptom. We assure you that there is no harm associated with the procedures undertaken as those are practiced routinely in the optometric settings.

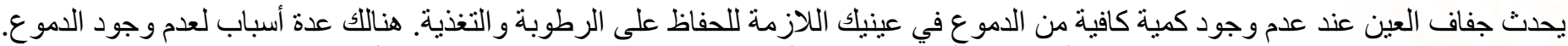

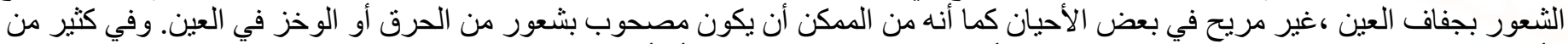

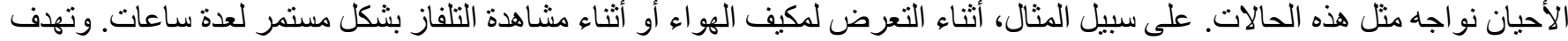

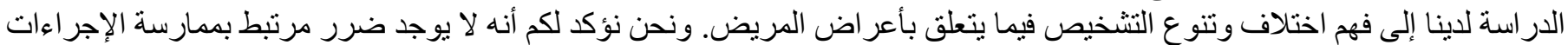

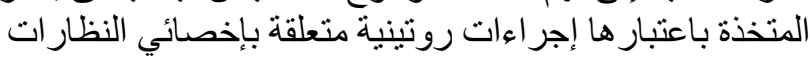


Would you kindly answer the following questionnaire as I need it in my research project. Please read the instructions carefully and start answering the questions given. If you agree to participate in the study without any opposition. Please sign below.

$$
\begin{aligned}
& \text { أرجو التكرم بالإجابة على الاستبيان التالي و أنا في حاجة إليها في مشروع بحثني. الرجاء قر اءة التعليمات بعناية والبدء في الإجابة على الأسئلة } \\
& \text {. المحددة. إذا و افقت على المشاركة في الدر اسة دون أي معارضة. الرالية الرجاء التوقيع في الأسفل }
\end{aligned}
$$

Signature:

\section{MCMONNIES' DRY EYE QUESTIONNAIRE WITH SCORING SCHEME'}

Please circle either : female/male. Age: less than 25years/25-45years/more than 45 years.

يرجى وضع دائرة إما : أناث/ذكور .العمر: أقل عن 25 سنه / 25-45 سنه / أكثر من 45 سنه

Currently wearing : no contact lenses / hard contact lenses / soft contact lenses.

ترتدي حالياً: لا نرتدي العدسات اللاصقة / العدسات اللاصقة الصلبة / العدسات اللاصقة اللينة.

Please answer the following by underlining the responses most appropriate to you.

الرجاء أجب على الاتي من خلال تسليط الضوء على الاستجابات الأكثر ملاءمة للك.

1. Have you ever had drops prescribed or other treatment for dry eyes?

Yes (2) / No(1) / Uncertain (0)

هل سبق للك ان استخدمت قطور أو علاج لجفاف العين؟1. إن

0) (نعم (2) / لا (1) / غير مؤكد )

2. Do you ever experience any of the following dry eye symptoms?

1 Soreness(1) 2 Scratchiness (1) 3 Dryness (1) 4 Grittiness (1) 5 Burning

هل سبق للك أن و اجهت أب من أعر اض جفاف العين التالية ؟.2

التقرح (1) 2 الخشونة (1) 3 جفاف (1) 4 رمل (1) 5 حرق 1

3. How often do your eyes have these symptoms?(underline)

Never (0) Sometimes (1) Often (2) Constantly (3)

3. (اهي غالبية شعورك بهذه الأعر اض في عينيك ؟ (أكد )

(أبدا (0) في بعض الأحيان (1) في كثير من الأحيان (2) باستمر ار (3)

4. Are your eye usually sensitive to cigarette smoke, smog, air conditioning, or central heating ? Yes (2) No

(0) Sometimes (1)

هل عينك عادة حساسة لدخان السجائر، و الدخان، وتكييف الهو اء، أو التدفئة المركزية؛؟ 
1) (2) (2) لا (2) في بعض الأحيان (2)

5. Do your eyes become very red and irritated when swimming?

Not applicable (0) Yes (1) No (0) Sometimes (1)

5ل تصبح عينيك حمر اء جدا وتكون منزعج عند السباحة؟

1) (لا ينطبق (0) نعم (1) لا (0) في بعض الأحيان

6. Do you take antihistamine tablets (1) or use antihistamine eye drops(1), diuretics (1) (Fluid tablet )

6. (هل تتناول أقر اص مضادات الهيستامين (1) أو تستخدم مضادات الهيستامين كقطور للعين (1)، مدر ات البول (1) (القرص السائل

7. Do you suffer from arthritis ? Yes (2) No (0) Uncertain (1)

7. (هل كنت تعاني من التهاب المفاصل؟ نعم (2) لا (0) غير مؤكد (1)

8. Do you experience dryness of the nose, mouth, throat?

Never (0) sometimes (1) Often (2) Constantly (3)

هل و اجهت جفاف الأنف و الفم و الحلق ؟8

(أبدا (0) في بعض الأحيان (1) في كثير من الأحيان (2) باستمر ار (3)

9. Do you suffer from thyroid abnormality?

Yes(2) No (0) Uncertain

9ل تلعاني من خلل الغدة الدرقية؟ نعم (2) لا (0) غير مؤكد

10. Are you known to sleep with your eyes partly open?

Yes (2) No(0) Sometimes (1)

هل أنت معروف بنومك و عينيك مفتوحه جزئياً.

1) (نعم (2) لا (0) في بعض الأحيان

11. Do you have eye irritation as you wake from sleep ?

Yes (2) No (0) Sometimes (1)

11. هل تهيج عينيك عندما تستيقظ من النوم؟

1) (نعم (2) لا (0) في بعض الأحيان

Thank you for taking the time to complete the questionnaire.

أشكركم على أخذ الوقت لاستكمال الاستبيان. 
Total score for answers 1 to 11 :

\begin{tabular}{|l|l|l|}
\hline \multicolumn{1}{|c|}{ Gender } & \multicolumn{1}{c|}{ Age } & Score \\
\hline Male or female & Under 25 & 0 \\
\hline Male & $25-45$ & 1 \\
\hline Female & $25-45$ & 3 \\
\hline Male & Over 45 & 2 \\
\hline Female & Over 45 & 6 \\
\hline
\end{tabular}

A score of over 20 is indicative of dry eye, while a total score of between 10 and 20 is suggestive of borderline dry eye disease.

\section{Comments:}

